\title{
EMPODERAMENTO COMUNITÁRIO NA BUSCA DE SOLUÇÕES PARA O PROBLEMA DA AUSÊNCIA DE SANEAMENTO BÁSICO NO BAIRRO DE PENEDO, MUNICÍPIO DE ITATIAIA, RIO DE JANEIRO, BRASIL
}

\author{
COMMUNITY EMPOWERMENT IN THE SEARCH FOR SOLUTIONS TO THE PROBLEM \\ OF THE LACK OF BASIC SANITATION IN THE NEIGHBORHOOD OF PENEDO, \\ MUNICIPALITY OF ITATIAIA, RIO DE JANEIRO, BRAZIL
}

\section{Bruno Luiz Silveira de Castro $^{1}$}

RESUMO: A escola sempre foi o reflexo de uma comunidade, não é à toa que o Projeto Político Pedagógico - PPP deve ser baseado na realidade local e na formulação de ações que visem sanar os problemas enfrentados pela comunidade. Entretanto, a realidade mesma que representada nos PPP parece não entrar nas salas de aula. A contextualização se faz necessária para o entendimento da realidade da comunidade. A cidade de Itatiaia, a qual encontra-se no estado do Rio de Janeiro carece de ações para um saneamento básico adequado. Através de ações de imersão da comunidade na realidade local é que se pode buscar ações que cobrem das autoridades competentes a resolução deste problema sanitário. O bairro de Penedo, alvo deste, é um bairro de apelo turístico e recebe pessoas de várias regiões, cidades, estados e países, mesmo apresentando este problema crônico. O objetivo proposto é demonstrar a importância da Educação Básica no entendimento da necessidade de um saneamento básico de qualidade partindo do princípio da alfabetização e letramento científico para promover uma leitura de mundo e assim fazer com que a comunidade empodere-se na busca de soluções e exerça de fato seu papel de cidadão.

Palavras-chave: Alfabetização científica. letramento científico. Empoderamento comunitário.

ABSTRACT: The school has always been the reflection of a community, it is not without reason that the Political Pedagogical Project - PPP must be based on the local reality and on the formulation of actions that aim to solve the problems faced by the community. However, the same reality that is represented in PPPs does not seem to enter the classrooms. Contextualization is necessary to understand the reality of the community. The city of Itatiaia, located in the state of Rio de Janeiro, lacks actions for adequate basic sanitation. Through actions of immersion of the community in the local reality, it is possible to seek

1 Estudante do Programa de Pós-Graduação em Práticas de Desenvolvimento Sustentável - Mestrado Profissional - Instituto de Florestas - Universidade Federal Rural do Rio de Janeiro. E-mail: bruno.silveiracastro@hotmail.com. 
actions that demand solutions to solve this health problem from the competent authorities. The district of Penedo, subject of this study, is a neighborhood of tourist appeal and receives people from several regions, cities, states and countries, even though it presents this chronic problem. The proposed objective is to demonstrate the importance of Basic Education to understanding the need for quality basic sanitation based on the principle of literacy and scientific literacy to promote a better comprehension of the world and, thus, empower the community to search for solutions and to exercise in fact their role as citizens.

Keywords: Scientific literacy. scientific literacy. community empowerment.

\section{INTRODUÇÃO}

As parasitoses representam importantes problemas de saúde pública e constituem uma ameaça constante à saúde e ao bem-estar da população, causando perdas econômicas com a redução da produtividade. Os problemas decorrentes das parasitoses afetam principalmente crianças na fase escolar, causando alterações relacionadas ao estado nutricional, crescimento e funções cognitivas. Diversos fatores, como precárias condições socioeconômicas, escolarização deficiente e falta de saneamento básico, contribuem para este quadro.

De acordo com Finger (2012), o parasitismo é classificado como uma relação prolongada e íntima entre o hospedeiro e o parasito, sendo somente o parasita é beneficiado por esta relação interespecífica. Esses parasitos apresentam ciclos de vida divididos em períodos com hospedeiros humanos, de vida livre no ambiente ou hospedando outros animais, sendo a infecção humana mais comum em crianças, através da via oral-fecal, tendo a água e alimentos contaminados como os principais veículos de transmissão (TOSCANI et al., 2007; COSTA; FERREIRA; MEIRELLES, 2015).

O saneamento básico é a principal medida que causa maior impacto sobre algumas das principais doenças humanas, inclusive as endoparasitoses (PEREIRA et al., 2010). A partir de dados obtidos juntos ao Instituto de Pesquisa Econômica Aplicada (IPEA) e Instituto Brasileiro de Geografia e Estatística (IBGE) por volta de 34,5 milhões de brasileiros que residem em áreas urbanas não têm acesso à coleta de esgoto (CARNEIRO, 2008), I6\% dos brasileiros não têm acesso à água tratada e mais de ıoo milhões de cidadãos buscam 
alternativas diferentes para suprirem as suas necessidades devido à ausência do poder público (VELASCO, 2020).

Itatiaia é uma cidade localizada no médio-paraíba no Estado Federativo do Rio de Janeiro e que apresenta uma variedade enorme de belezas naturais, como, por exemplo, o Pico das Agulhas Negras - a montanha mais alta do Brasil, o bairro Penedo - a pequena Finlândia, Maromba e Maringá. Economicamente, a cidade está em expansão com a chegada de várias empresas como Hyundai, Land Rover - Jaguar, Arno e entre outras.

Por outro lado, a cidade carece de infraestrutura, o seu crescimento desordenado é complacente com sucessivas invasões de áreas particulares e da União, o que faz com que o poder público sempre esteja tentando mitigar os impactos das invasões, como promover arruamentos, coleta de lixo e esgoto, aumento do alcance do transporte escolar, fornecimento de energia elétrica e água encana, mas não tratada.

Somente 2.075 habitantes (de uma população de 29.448 habitantes) são atendidos com esgotamento sanitário recolhido, sendo que nenhuma percentagem do volume do esgoto recolhido é tratada (GUEDES; PERTEL; ACSELRAD, 2018). A distribuição de água não é cobrada e não há estação de tratamento de água - ETA (ALMEIDA, 2005), o que leva a períodos de surtos de infecções de endoparasitoses.

Apesar do bairro Penedo ter um apelo turístico fortíssimo, carece de medidas simplórias quanto ao saneamento básico digno. É comum a grande maioria dos turistas julgarem que Penedo é uma cidade, assim como Campos do Jordão é uma cidade. Entretanto, é um bairro. Um bairro que pertence ao município de Itatiaia. Na região onde encontra-se Penedo, existem outros bairros carentes que afloram as desigualdades sociais e refletem de fato a ausência do saneamento básico, como, por exemplo, os bairros: África I, África II e Jambeiro.

Contudo, o objetivo proposto é demonstrar a importância da Educação Básica no entendimento da necessidade de um saneamento básico de qualidade. Através da alfabetização e letramento científico é que a comunidade escolar terá a percepção da realidade precária que se encontram (CHASSOT, 2003). Ter conhecimento da realidade promove 
ações para busca de soluções. Imergir a comunidade na realidade proporciona um emergir cheio de atitudes da mesma (FREIRE, 2019). Através do conhecimento situacional de grupos ou indivíduos é que há o empoderamento comunitário e por ele ocorre a busca do bem comum para o coletivo (GOHN, 2004; BAQUERO, 2012).

\section{METODOLOGIA}

Tratar-se-á de uma revisão bibliográfica (CERVO, BERVIAN, SILVA, 2007) para ter sustentação da ideia principal do texto. Somente ensinar o que é saneamento básico não é suficiente. Há de se inserir a comunidade na situação real em que se encontra. Juntamente com ela perceber e entender as necessidades de mudanças nas políticas sanitárias do município em questão.

Identificar que a ausência de informação pode promover a infecção por doenças que dificilmente causam morte, mas que trazem sérios riscos a população (NEVES et al., 2005; ANDRADE et al., 2010), e que juntamente com o pouco interesse do poder público acarreta mais problemas de saúde pública (KUNZ et. al., 2008; WHO, 2012).

Esta relação parasitose intestinal com ausência de saneamento básica é uma questão socioambiental, assim como a ausência de um plano de governo que promova a destinação e tratamento do lixo é uma questãos socioambential, a ausência de estação de tratamento da água (ETA) e estação de tratamento de esgoto (ETE) são problemas socioambientais que carecem de medidas urgentes para saná-los.

\section{DISCUSSÃO}

O saneamento básico é constituído por um conjunto de ações que visam entregar salubridade ambiental. Os sistemas de saneamento envolvem diversas soluções, por exemplo, para o abastecimento de água tratada, recolhimento e tratamento dos esgotos, essas atividades devem ter qualidade e quantidade suficientes para promoção da saúde pública e controle da poluição ambiental, segundo Ministério da Saúde (BRASIL, 2004).

Vale ressaltar que água potável e saneamento básico encontram-se como um dos 
objetivos do desenvolvimento sustentável - ODS 6 para a Agenda 2030 o qual diz: “Assegurar a disponibilidade e gestão sustentável da água e saneamento para todos” (AGENDA 2030, 2015).

Corroborando o supracitado, a Organização Mundial da Saúde - OMS estipula que possam ocorrer mais de 200 milhões de casos anuais. As enteroparasitoses são frequentes no Brasil, especialmente entre as crianças e uma das principais consequências é baixa capacidade de concentração e dificuldades no aprendizado (KUNZ et al., 2008) podendo apresentar também graves consequências sobre o estado de desenvolvimento físico e mental, principalmente das criança, sendo que a maioria dessas crianças acometidas encontram-se em período escolar (WHO, 2012).

Para Zaiden et al. (2008), a resolução deste problema de saúde pública está inserida na necessidade de conhecimento da epidemiologia das endoparasitoses, principalmente as endêmicas. Através do currículo, o educador pode promover metodologias que façam com que os alunos tenham a compreensão da vida e do seu papel nela e entenda que os sistemas estão organizados e integrados através de interação com o ambiente (BRASIL, 2006).

A escola tem grande importância na divulgação da epidemiologia das endoparasitoses (COSTA; FERREIRA; MEIRELLES, 2015). Por isso, a educação é a medida preventiva efetiva e tem sido utilizada por vários pesquisadores no processo de prevenção às parasitoses (MELLO et al., 1988; PEDRAZZANI et al., I990; FERREIRA; ANDRADE, 2005).

Costa, Ferreira e Meirelles (2015) apontam lacunas na construção do conhecimento acerca da relação da água com a transmissão de algumas doenças, sugerindo necessidade de discussão sobre o tema entre os alunos.

Entretanto, Silva e Leda (2012) afirmam ser um paradoxo ensinar hábitos saudáveis de higiene e não ter o fornecimento adequado de água potável para que os alunos possam, por exemplo, lavar as mãos antes de se alimentarem. Além do contato direto com a água contaminada, outra forma de contágio é através da alimentação que muitas vezes é manuseada por quem não tem conhecimento das formas de transmissão (SILVA et al., 2015) ou é lavada com água contaminada ou já se encontram contaminados por falta de fiscalização 
da higiene desses produtos (CANTOS et al., 2004).

Contudo, somente pela alfabetização e letramento científico é que se pode obter os pilares necessários para mudança do comportamento da comunidade. Pois, a alfabetização e letramento científico privilegia uma educação mais comprometida com a realidade. Promove um entendimento de mundo. Ler a natureza através da sua escrita (CHASSOT, 2003).

Segundo Chassot (2003) através do entendimento da natureza que é possível projetar ações e prever comportamentos. O entendimento do mundo conduz a qualidade de vida, pois o conhecimento das possibilidades e alternativas foi percebido pelo alfabetizado cientificamente. Logo, é deverás importante considerar a ciências com linguagem necessária para promoção do conhecimento do mundo. Assim, pela alfabetização e letramento científico ocorre a inclusão social do cidadão.

A Constituição Federal de I988 (BRASIL, 1988) no seu artigo 5.ํㅡ, versa sobre a igualdade de todos perante a Lei, a princípio parecer ser o mais justo. Entretanto, tratar todos por igual não é justiça, pois as caminhadas são diferentes, as oportunidades são escassas para maioria da população. O viés de ação é outro, as políticas precisam atender as necessidades dos diferentes grupos nas suas diferentes realidades.

E a partir da alfabetização e letramento científico, como disse Chassot (2003) os indivíduos podem exercer a sua cidadania. Cidadania não no modelo republicano (PINHÃO; MARTINS, 2016), mas sim uma cidadania de fato, fruto do empoderamento comunitário proposto pela imersão dos grupos ou indivíduos na situação em que se encontram (GOHN, 2004; BAQUERO, 2012).

Para Gohn (2004) e Baquero (2012) o empoderamento se faz através de ações participativas da comunidade em busca do conhecimento situacional. A partir do momento em que o grupo percebe a realidade através da leitura da natureza é possível promover ações de libertação e que visem atingir objetivos para melhorar a qualidade de vida de todos os envolvidos.

É importante salientar que, assim como disse Freire (2019), não há um salvador da pátria nessa dinâmica participativa, ninguém pode se considerar como o detentor do 
conhecimento, pois o entendimento da realidade difere para cada um. O que há de se fazer é imergir com a comunidade na realidade local para que todos, até a liderança revolucionária, entenda, utilizando o processo de decodificação da realidade, analisando parte por parte e depois codificando tudo novamente para então, assim, todos buscarem melhores formas de ação durante a emersão da realidade, agora, cientes do problema, passíveis de conquista.

\section{CONSIDERAÇÕES FINAIS}

Para a busca de uma participação mais efetiva da comunidade no dia a dia se faz necessária que todos estejam cientes da realidade. A promoção da cidadania do indivíduo se dá pela exercimento da mesma. Entretanto, a perceção de cidadania num modelo Republicano é errada, visto as necessidades da comunidade, promover que todos têm direitos iguais não fundamenta política alguma, pois, às necessidade são diferentes, a caminhada é diferente, não há igualdade na busca pelo melhor.

É fundamentalmente importante inserir a comunidade escolar na real situação sanitária do município de Itatiaia, mais especificamente de Penedo. A imersão na realidade é imprescindível, é crucial que a decodificação da realidade para posteriormente a codificação, só assim, todos os envolvidos poderão exercer de fato o seu papel de cidadão, numa cidadania pautada no conhecimento da realidade.

Para haver o empoderamento comunitário os atores devem ser envolvidos através de metodologias participativas, as quais são pautadas em ações que visam atingir determinados objetivos que foram definidos pelo coletivo. Só assim, no comprometimento daqueles grupos ou indivíduos considerados desfavorecidos é que poderão conquistar o seu papel de cidadãos e partirem em busca dos seus interesses comuns junto ao poder público.

\section{REFERÊNCIAS BIBLIOGRÁFICAS}

AGENDA 2030. 2015. Plataforma Agenda 2030: Acelerando as transformações para a Agenda 2030 no Brasil. Disponível em: http://www.agenda2030.com.br/. Acesso em: 9 maio 202I.

ALMEIDA, R. Em Itatiaia, o consumo de água é livre e o desperdício é grande. Disponível 
em: https://oglobo.globo.com/rio/em-itatiaia-consumo-de-agua-livre-desperdicio-grandeI5215887.n . Acesso em: is jun. 2020.

ANDRADE, E. C. et al. Parasitoses Intestinais: Uma revisão sobre os seus aspectos sociais, epidemiológicos, clínicos e terapêuticos. Rev. APS, Juiz de Fora, v. 13, n. 2, p. 231-240, abr./jun. 2010.

BAQUERO, R. V. A. Empoderamento: instrumento de emancipação social? - Uma discussão conceitual. Revista Debates, Porto Alegre, v. 6, n. I, p. 173 - I87, jan. - abr. 2013.

BRASIL. Constituição da República Federativa do Brasil. Brasília, DF: Senado Federal, 1988.

BRASIL. Ministério da Saúde. Organização Pan-Americana da Saúde. Avaliação de impacto na saúde das ações de saneamento: marco conceitual e estratégia metodológica. Brasília: Ministério da Saúde, 2004. II6 p.

BRASIL. Orientações Curriculares para o Ensino Médio. Ciências da natureza, matemática e suas tecnologias. Ministério da Educação, Secretaria de Educação Básica, Brasília, v. 02, 137 p. 2006.

CARNEIRO, F.- 2008. Brasil tem 34,5 milhões de pessoas sem esgoto em áreas urbanas: Percentual de negros sem acesso a saneamento é quase o dobro do de brancos. Outras 13,2 milhões de pessoas vivem em cortiços nas cidades, mostra Ipea. Disponível em: http://gi.globo.com/Noticias/Brasil/o,,MUL583664-5598,oo$\mathrm{BRASIL}+\mathrm{TEM}+\mathrm{MILHOES}+\mathrm{DE}+\mathrm{PESSOAS}+\mathrm{SEM}+\mathrm{ESGOTO}+\mathrm{EM}+\mathrm{AREAS}+\mathrm{URBANAS}$. html. Acesso em: 15 jun. 2020.

CANTOS, G. A. et al. Estruturas parasitárias encontradas em hortaliças comercializadas em Florianópolis, Santa Catarina. Newslab. v. 66. p. 154-163. 2004.

CERVO, A. L.; BERVIAN, P.A.; SILVA, R. Metodologia científica. 6 ed. São Paulo: Pearson Prentice Hall, 2007.

CHASSOT, A. Alfabetização científica: uma possibilidade para inclusão social. Revista Brasileira de Educação. p. 89 - Ioo. jan./fev./mar./abr. 2003.

COSTA, E. C. P.; FERREIRA, C. P.; MEIRElleS, R. M. S. Percepções dos alunos do ensino fundamental da rede pública de ensino acerca das parasitoses intestinais. $\mathrm{X}$ Encontro Nacional de Pesquisa em Educação em Ciências - X ENPEC Águas de Lindoia, SP. nov. 2015 .

FERREIRA, G. R.; ANDRADE, C. F. S.. Alguns aspectos socioeconômicos relacionados a parasitoses intestinais e avaliação de uma intervenção educativa em escolares de Estiva Gerbi, 
SP.Revista da Sociedade Brasileira de Medicina Tropical. Uberaba, v. 38, p. 402-405, 2005. Disponível em: http://www.scielo.br/pdf/rsbmt/v38n5/ao8v38n5.pdf. Acesso em: is jun. 2020

FINGER, E. - A termodinâmica como princípio motriz do sistema imune. Einstein. v.ıo, n. 3, São Paulo. jul-set. 2012.

FREIRE, P. Pedagogia do oprimido. 7r ed. Rio de Janeiro/São Paulo: Paz e Terra, 2019.

GOHN, M. G. Empoderamento e participação da comunidade em políticas sociais. Saúde e Sociedade. v. 13, n. 2, p. 20 - 31. maio - ago. 2004.

GUEDES, R. B.; PERTEL, M.; ACSELRAD, M. V. Cobrança pelo uso da água bruta no estado do rio de janeiro e demanda por investimentos para ampliação do atendimento em coleta e tratamento de esgotos nas bacias fluminenses afluentes ao Rio Paraíba do Sul. III Simpósio de Recursos Hídricos da Bacia do Rio Paraíba do Sul. Juiz de Fora - MG. Ago. 2018.

KUNZ, J. M. O. et al. Parasitas intestinais em crianças de escola municipal de Florianópolis, SC. Educação ambiental e em saúde. Biotemas. 2008; v. 21, p. 157-62.

MELlO, D. A. et al. Helmintoses intestinais: Conhecimentos, atitudes e percepção da população. Revista de Saúde Pública de São Paulo. v. 22, p. 140-I49, 1988.MELLO, D. A. et al. Helmintoses intestinais: Conhecimentos, atitudes e percepção da população. Revista de Saúde Pública de São Paulo. v. 22, p. I40-I49, 1988.

NEVES, D. P. et al. Parasitologia Humana. Ir. ed. São Paulo: Editora Atheneu, 2005. 498 p.

PEDRAZZANI, E. S. et al. Helmintoses intestinais. Revista de Saúde Pública de São Paulo. v. 22, p. 384-389, 1988.

PEREIRA, V. V. et al. Avaliação de parasitoses intestinais, estado nutricional e indicadores sociais em alunos de quatro escolas do ensino fundamental público da cidade de Divinópolis Minas Gerais - Brasil. Neotropical Helminthology, v. 4, n. 2, p. 149-157, 2010.

PINHÃO, F.; MARTINS, I. Cidadania e ensino de ciências: questões para o debate. Ens. Pesqui. Educ. Ciênc., Belo Horizonte, v. I8, n. 3, p. 9 - 29, dez. 2016.

SILVA, A. O. et al. Epidemiologia e prevenção de parasitoses intestinais em crianças das creches municipais de Itapuranga-GO. Rev. Facul. Mont. Bel. v. 8, n. I, p. I-17, 2014.

SILVA, T. V. DA; LEDA, L. R. Intervenções educativas sobre parasitoses intestinais: aplicação de um jogo para alunos do ensino fundamental. Saúde \& Amb. Rev., Duque de 
Caxias. v. 5, n. 2, p. 23-37, jul-dez. 2012.

TOSCANI, N. V. et al. Desenvolvimento e análise de jogo educativo para crianças visando à prevenção de doenças parasitológicas. Interface - Comunic., Saúde, Educ., v. II, n. 22, p. 28I- 294, maio - ago. 2007.

VELASCO, C. 2020. Raio X do saneamento no Brasil: $16 \%$ não têm água tratada e 47\% não têm acesso à rede de esgoto. Índices do setor apontam que a universalização dos serviços ainda está distante. Novo marco legal do saneamento básico deve ser votado nesta quarta-feira (24) pelo plenário do Senado. Disponível em: https://gr.globo.com/economia/noticia/2020/06/24/raio-x-do-saneamento-no-brasilI6percent-nao-tem-agua-tratada-e-47percent-nao-tem-acesso-a-rede-de-esgoto.ghtml. Acesso em I9 dez. 2020.

WORLD HEALTH ORGANIZATION. Distribution of soil-transmitted helminthiases and proportion of children (agedI-I4 years) in each endemic country requiring preventive chemotherapy for the diseases. 2012.

ZAIDEN, M. et al. Epidemiologia das parasitoses intestinais em crianças de creches de Rio Verde- GO. Medicina (Ribeirão Preto. Online) 30 jun. 2008. v. 4I, n. 2, p. I82-187. Disponível em: http://www.revistas.usp.br/rmrp/article/view/265. Acesso em: I8 out. 2019. 